\title{
Image Reconstruction Under Multiplicative Speckle Noise using Total Variation
}

\author{
Manya V. Afonso, João M. R. Sanches \\ Instituto de Sistemas e Robótica, Instituto Superior Técnico, Lisbon, Portugal \\ \{mafonso,jmrs\}@isr.ist.utl.pt
}

\begin{abstract}
In this paper, we present a method for reconstructing images or volumes from a partial set of observations, under the Rayleigh distributed multiplicative noise model, which is the appropriate algebraic model in ultrasound (US) imaging. The proposed method performs a variable splitting to introduce an auxiliary variable to serve as the argument of the total variation (TV) regularizer term. Applying the Augmented Lagrangian framework and using an iterative alternating minimization method leads to simpler problems involving TV minimization with a least squares term. The resulting Gauss Seidel scheme is an instance of the Alternating Direction Method of Multipliers (ADMM) method, for which convergence is guaranteed. Experimental results show that the proposed method achieves a lower reconstruction error than existing methods.
\end{abstract}

Keywords: despeckling, image reconstruction, multiplicative noise, ultrasound, convex optimization, total variation, iterative algorithms

\section{Introduction}

Images acquired using any modality need to be denoised before further processing or analysis. The problem of estimating the image from the noisy and possibly incomplete observations is an ill-posed one [1], necessitating regularization or some assumption on the nature of the image. The statistical distribution of the noise and the algebraic observation model that leads to the image formation depends on the physics of the modality. Further, depending on hardware and sensing limitations as well as transmission errors, the image may need to be reconstructed from a set of partial observations. 
This is also the case in the sampling/acquisition methodology known as compressive sensing $[2,3]$ wherein the image needs to be reconstructed from an undersampled set of incoherent observations.

Several methods exist to solve the denoising and reconstruction problems for the classical additive and Gaussian noise model, using non-smooth regularization such as total variation (TV) $[4,5,6]$ which encourages the solution to be piece-wise smooth. Recent methods have focussed on the augmented Lagrangian/alternating direction method of multipliers (AL/ADMM) method $[7,8]$ to solve the convex optimization formulations for these TV regularized problems $[9,10,11]$ because of its computational speed. In this paper, we propose a TV and ADMM based method for image denoising and reconstruction from partial observations for the case when the noise is multiplicative and Rayleigh distributed, which is the model in Ultrasound (US) imaging for the radio frequency $(\mathrm{RF})$ envelope image [12].

Ultrasound has emerged as a popular medical imaging modality in a number of medical imaging applications because of its low cost, wide reach, flexibility, lack of radiation, and intra-operability $[13,14,15]$. Because 2D US images are acquired as slices representing a thin plane from the volume, it is difficult to reproduce for follow-up, i.e., image at the exact location again. Therefore three dimensional (3D) US imaging is being increasingly used for characterizing diseases such as carotid atherosclerosis, requiring a 3D volume to be reconstructed from a series of $2 \mathrm{D}$ slices. The slices can be acquired mechanically in a predetermined manner, or freehand wherein the user can manually position and orient the probe. It has also been reported that segmentation and classification based on 3D US has advantages compared to those based on 2D $[16,17]$.

\subsection{Related Works}

For the additive and Gaussian noise case, solvers for 2D and 3D reconstruction from partial data using TV regularization include the Sparse Reconstruction by Separable Approximation [18], (Constrained) Split Augmented Lagrangian Shrinkage Algorithm [11, 19], split Bregman method [10], Fast TV deconvolution (FTVd) [9], and the Nesterov method based solvers, mxTV [20], and NESTA [21]. Sparse MRI [22] for MRI reconstruction also uses a TV regularizer term.

A despeckling method for US images called Rayleigh Log-Euclidean Total Variation (RLTV) was proposed in [23, 24], in which a logarithmic compression was used on the image, and then a TV regularizer term was applied 
on the transformed image. The resulting convex optimization problem was solved iteratively using Newton's method. The same denoising formulation was solved in multiplicative image denoising by augmented Lagrangian (MIDAL) method [25] in the context of Synthetic Aperture Radar (SAR) images, but solved using an AL/ADMM method. MIDAL also does not use a logarithmic compression. Another TV based denoising method for gamma distributed multiplicative speckle noise is the variational formulation based on $m^{\text {th }}$ root transformation called linearized proximal alternating minimization algorithm (LPAMA/mV) [26, 27]. This method was proposed for the application of multi-look SAR images. The Nakagami distribution was assumed as the statistical model in the denoising method presented in [28]. A denoising method was presented in [29], assuming that the multiplicative noise (in natural images) was one-sided exponentially distributed, and with an $\ell_{1}$ data fidelity term.

A variational model for deblurring under multiplicative noise was proposed in [30], which uses a quadratic penalty term and is strictly convex under mild conditions. The formulation is solved using a primal-dual algorithm.

The above listed methods solve the despeckling problem when there is no loss of pixels. To solve the harder problem of image reconstruction, in [24], the authors first perform a voxel interpolation over the grid to obtain a noisy image without missing pixels, and then apply RLTV to despeckle it.

Interpolation algorithms such as the Pixel Nearest Neighbor (PNN) [31], Voxel Nearest Neighbor (VNN) [31], Pixel-Based Interpolation with Distance Weighting (PBM-DW) [32], etc do not use any regularization or apriori information about the volume to be reconstructed. A comprehensive review of interpolation methods for US reconstruction can be found in [14].

Other non TV based methods for 3D US reconstruction have been reported in literature. These include the Cyclic Regularized Savitzky-Golay (CRSG) filter method [33] which estimates unobserved voxels through a local 3D least squares polynomial fitting. Results reported in this work showed that CRSG was able to obtain a lower normalized reconstruction error (0.032) than PNN-DW (0.047) in 3D synthetic experiments. Others such as [13] perform an interpolation and coordinate mapping over each unobserved voxel. Spline interpolation to connect regions across observed slices acquired freehand has also been proposed [34]. In this work, results were reported for different conditions of the carotid artery (normal or with plaque stenosis), without comparison with existing methods. A despeckling filter based on 
anisotropic diffusion without a linear approximation (DPAD) was proposed in [35] for denoising and separating the speckle component.

\subsection{Contributions}

In this paper, we extend the TV regularized despeckling formulation from [23] [25] to the more general problem of estimating the image from a partial set of noisy pixels. This is a more difficult and ill-posed [1] problem than denoising, because some pixel/voxel values are unknown. This is a relevant problem from the point of view of reconstructing a 3D volume from a partial set of acquired $2 \mathrm{D}$ slices. We solve the resulting convex problem using an AL/ADMM approach which leads to an alternating minimization in which at every iteration a sequence of simpler problems has to be solved. The proposed method for reconstruction is a more general formulation of the method for solving the denoising problem alone. We test the proposed method with synthetic data simulating both linear mechanical and random freehand scanning, as well as real US images. Preliminary results were presented in [36], which showed that the proposed method is more accurate than interpolation methods, and is faster than all methods except the Pixel Nearest Neighbor $(\mathrm{PNN})$ interpolation which is the crudest interpolation technique. In this paper, we compare our method against PNN interpolation followed by despeckling methods which take into account the statistical model. Synthetic experiments show that the proposed method achieves a lower mean square error than existing methods.

In section 2, we formulate the optimization problems to be solved for estimating the despeckled image, with and without missing data. We present the proposed approach for solving the denoising and reconstruction problems in section 3. In Section 4, we present experimental results on 2D and $3 \mathrm{D}$ reconstruction, with synthetic examples and real US images. Section 5 concludes the paper.

\section{Problem Formulation}

The image is represented as a vector, say, in lexicographic ordering, as $\mathbf{x} \in \mathbb{R}^{n}$, where $n$ is the number of pixels or voxels. When there is no loss of pixels, the dimensionality of the observed image $\mathbf{y}$ is the same as that of $\mathbf{x}$. Each element of $\mathbf{y}$ is the product of the corresponding element from $\mathbf{x}$ and the corresponding element from the noise field $\eta$. The observation model is 
therefore the element-wise multiplication,

$$
\mathbf{y}=\mathbf{x} \cdot \eta
$$

In the case of partial observations, the number of elements of $\mathbf{y}$ is less than the size of $\mathbf{x}$. This is the case in the problem of inpainting, wherein pixels damaged or lost because of transmission errors have to be estimated $[37,38,39]$. The acquisition methodology of compressive sensing also involves observing an incomplete set of incoherent observations to speed up and simplify the sensing process and hardware [22]. When the number of observed pixels is $m<n$, we model the observation process as a multiplication of $\mathbf{x} \in \mathbb{R}^{n}$ by a linear operator $\mathbf{A} \in \mathbb{R}^{m \times n}$,

$$
\mathbf{y}=(\mathbf{A x}) \cdot \eta
$$

In this case, $\mathbf{y}, \eta \in \mathbb{R}^{m}$. The matrix $\mathbf{A}$ maps a pixel or voxel in the grid to a pixel in the set of observed slices, and discards the pixels or voxels in $\mathbf{x}$ which don't correspond to a pixel in $\mathbf{y}$. Hence, the matrix $\mathbf{A}$ is essentially the $n \times n$ identity matrix with $n-m$ rows (corresponding to non-observed voxels) removed. The position and orientation of each slice must be known to construct the matrix A. For a denoising problem, i.e., when all elements are observed $(m=n)$, it is equal to the identity matrix $\mathbf{A}=\mathbf{I}$.

Assuming that the speckle field $\eta$ is Rayleigh distributed, when there are no missing observations the likelihood is,

$$
p(\mathbf{y} \mid \mathbf{x})=\prod_{i=1}^{n} \frac{y_{i}}{x_{i}} \exp \left(-\frac{y_{i}^{2}}{2 x_{i}}\right)
$$

where $x_{i}$ is the $i^{\text {th }}$ element of the vector $\mathbf{x}$. After logarithmic compression, this leads to the log-likelihood function,

$$
E(\mathbf{y}, \mathbf{x})=-\log (p(\mathbf{y} \mid \mathbf{x}))=\sum_{i=1}^{n}\left(\frac{y_{i}^{2}}{2 x_{i}}+\log x_{i}\right) .
$$

In [40], a logarithmic compression $\mathbf{f}=\log (\mathbf{x})$ is applied and a TV regularizer term is applied on the transformed variable, leading to the convex optimization problem,

$$
\min _{\mathbf{f}} \sum_{i}\left(\frac{y_{i}^{2}}{2} e^{-f_{i}}+f_{i}\right)+\frac{\lambda}{2} T V(\mathbf{f}),
$$


where as before, $f_{i}$ represents a pixel or voxel from the vector representation of the volume $\mathbf{f}$, and $\lambda>0$ is the regularization parameter. Because both the TV function and the log transformation are convex, adding the term $T V(\mathbf{f})$ to the data fidelity term from (4) leads to the same minimizer as applying the TV regularizer on $\mathbf{x}, T V(\mathbf{x})$. For more details see [41].

The isotropic TV semi-norm for the 2D case is defined as,

$$
\mathrm{TV}(\mathbf{f})=\sum_{\left(i_{1}, i_{2}\right)} \sqrt{\left(f_{i_{1}, i_{2}}-f_{i_{1}-1, i_{2}}\right)^{2}+\left(f_{i_{1}, i_{2}}-f_{i_{1}, i_{2}-1}\right)^{2}},
$$

where the coordinates $\left(i_{1}, i_{2}\right)$ are the row and column indices for a pixel. Similarly, in 3D the TV function is,

$$
\begin{aligned}
T V(\mathbf{f})= & \sum_{i}\left(\left(f_{i_{1}, i_{2}, i_{3}}-f_{i_{1}-1, i_{2}, i_{3}}\right)^{2}+\left(f_{i_{1}, i_{2}, i_{3}}-f_{i_{1}, i_{2}-1, i_{3}}\right)^{2}+\ldots\right. \\
& \left.+\left(f_{i_{1}, i_{2}, i_{3}}-f_{i_{1}-1, i_{2}, i_{3}-1}\right)^{2}\right)^{\frac{1}{2}}
\end{aligned}
$$

where the voxel indices are $\left(i_{1}, i_{2}, i_{3}\right)$. It must be noted that with vector representation, neighboring pixels or voxels do not necessarily occupy contiguous positions in the vector. Throughout the paper, we assume that a voxel indexed by the subscript $i$ corresponds to a set of coordinate values $\left(i_{1}, i_{2}, i_{3}\right)$.

We follow a similar approach for the case with partial observations (2). The likelihood function from (3) now changes to,

$$
p(\mathbf{y} \mid \mathbf{x})=\prod_{i=1}^{m} \frac{y_{i}}{(\mathbf{A} \mathbf{x})_{\mathbf{i}}} \exp \left(-\frac{y_{i}^{2}}{2(\mathbf{A} \mathbf{x})_{i}}\right)
$$

where $(\mathbf{A x})_{i}$ is the $i^{\text {th }}$ element of the sampled vector $\mathbf{A x}$. The associated log-likelihood function is therefore,

$$
E(\mathbf{y}, \mathbf{x})=-\log (p(\mathbf{y} \mid \mathbf{x}))=\sum_{i=1}^{m}\left(\frac{y_{i}^{2}}{2(\mathbf{A} \mathbf{x})_{i}}+\log (\mathbf{A x})_{i}\right)
$$

To formulate the problem of estimating the volume $\mathbf{x}$, given $\mathbf{y}$ with the statistical model (8), we apply the logarithmic transformation, $\mathbf{f}=\log (\mathbf{A x})$, and again formulate the estimation problem as the problem of minimizing the sum of the data fidelity term (9) and a TV regularizer term. In this case 
however, we cannot apply the TV term on $\mathbf{f}$ because it is not of the same dimensions as $\mathbf{x}$. Therefore, we define our variable as $(\mathbf{x}, \mathbf{f})$, resulting in the constrained convex problem,

$$
\min _{\mathbf{x}, \mathbf{f}} \sum_{i}\left(\frac{y_{i}^{2}}{2} e^{-f_{i}}+f_{i}\right)+\frac{\lambda}{2} T V(\mathbf{x}), \quad \text { subject to } \quad \mathbf{A x}=e^{\mathbf{f}} .
$$

This formulation is the generalized form of the TV regularized despeckling problem (5).

\section{Proposed Method}

In [40], the denoising problem (5) was solved using Newton's method. An ADMM based method was presented in [25] for solving a problem similar to (10) but without logarithmic compression, for the case of multiple look Synthetic Aperture Radar (SAR) imaging. The advantage of the AL/ADMM framework is that it requires simpler, separable problems to be solved at each iteration, leading to computational simplicity.

We first briefly review the concepts of variable splitting, augmented Lagrangian, and the alternating direction method of multipliers before applying them to our problems (5) and (10). Indeed, the denoising problem (5) is a particular case of the more general reconstruction problem (10), and the development of the respective methods will be shown in a unified manner.

\subsection{The $A L / A D M M$ Method}

Consider an unconstrained optimization problem in which the objective function is of the form,

$$
\min _{\mathbf{u} \in \mathbb{R}^{n}} f_{1}(\mathbf{u})+f_{2}(g(\mathbf{u})),
$$

where $g: \mathbb{R}^{n} \rightarrow \mathbb{R}^{d}$. Using variable splitting [42], a new variable, say $\mathbf{v}$, is created to serve as the argument of $f_{2}$, under the constraint that $g(\mathbf{u})=\mathbf{v}$. We now have the equivalent constrained problem

$$
\begin{array}{cl}
\min _{\substack{\mathbf{u} \in \mathbb{R}^{n}, \mathbf{v} \in \mathbb{R}^{d} \\
\text { subject to }}} & f_{1}(\mathbf{u})+f_{2}(\mathbf{v})=\mathbf{v} .
\end{array}
$$

The split-Bregman methods [10] attack the constrained problem using a Bregman iterative algorithm [8]. It has been shown that, when $g$ is a linear 
function, i.e., $g(\mathbf{u})=\mathbf{G u}$, the Bregman iterative algorithm is equivalent to the augmented Lagrangian method [8].

Consider the constrained optimization problem

$$
\begin{array}{cl}
\min _{\mathbf{z} \in \mathbb{R}^{n}} & E(\mathbf{z}) \\
\text { s.t. } & \mathbf{A z}-\mathbf{b}=\mathbf{0},
\end{array}
$$

where $\mathbf{b} \in \mathbb{R}^{p}$ and $\mathbf{A} \in \mathbb{R}^{p \times n}$. There are $p$ linear equality constraints. The so-called augmented Lagrangian function for this problem is defined as

$$
\mathcal{L}_{A}(\mathbf{z}, \boldsymbol{\lambda}, \mu)=E(\mathbf{z})+\boldsymbol{\lambda}^{T}(\mathbf{b}-\mathbf{A} \mathbf{z})+\frac{\mu}{2}\|\mathbf{A} \mathbf{z}-\mathbf{b}\|_{2}^{2},
$$

where $\boldsymbol{\lambda} \in \mathbb{R}^{p}$ is a vector of Lagrange multipliers and $\mu \geq 0$ is called the penalty parameter [43].

The so-called augmented Lagrangian method (ALM) [43], also known as the method of multipliers (MM) [44], [45], iteratively and alternatingly minimizes $\mathcal{L}_{A}(\mathbf{z}, \boldsymbol{\lambda}, \mu)$ with respect to $\mathbf{z}$, and $\boldsymbol{\lambda}$. The ALM/MM can be summarized as:

Algorithm $A L M / M M$

1. Set $k=0$, choose $\mu>0, \mathbf{z}_{0}$, and $\boldsymbol{\lambda}_{0}$.

2. repeat

3. $\quad \mathbf{z}_{k+1} \in \arg \min _{\mathbf{z}} \mathcal{L}_{A}\left(\mathbf{z}, \boldsymbol{\lambda}_{k}, \mu\right)$

4. $\quad \boldsymbol{\lambda}_{k+1}=\boldsymbol{\lambda}_{k}+\mu\left(\mathbf{b}-\mathbf{A} \mathbf{z}_{k+1}\right)$

5. $\quad k \leftarrow k+1$

6. until stopping criterion is satisfied.

The ALM/MM does not require $\mu$ to be updated to infinity to guarantee convergence to the solution of the constrained problem (13) [46, Chap. 9]. It can be verified that the terms added to $E(\mathbf{z})$ in the definition of the augmented Lagrangian $\mathcal{L}_{A}\left(\mathbf{z}, \boldsymbol{\lambda}_{k}, \mu\right)$ in (14) can be written as a single quadratic term in $\mathbf{z}$ plus a constant, leading to the following alternative form of the algorithm (which makes clear its equivalence with the Bregman iterative method [8]): 
Algorithm $A L M / M M$ (version II)

1. Set $k=0$, choose $\mu>0$ and $\mathbf{d}_{0}$.

2. repeat

3. $\quad \mathbf{z}_{k+1} \in \arg \min _{\mathbf{z}} E(\mathbf{z})+\frac{\mu}{2}\left\|\mathbf{A z}-\mathbf{d}_{k}\right\|_{2}^{2}$

4. $\quad \mathbf{d}_{k+1}=\mathbf{d}_{k}+\left(\mathbf{b}-\mathbf{A z}_{k+1}\right)$

5. $\quad k \leftarrow k+1$

6. until stopping criterion is satisfied,

where $\mathbf{d}$ is the vector of dual variables. With adequate initializations, the ALM/MM generates the same sequence as a proximal point algorithm applied to the Lagrange dual of problem (13) [47]. Moreover, the sequence $\left\{\mathbf{d}_{k}\right\}$ converges to a solution of this dual problem and all cluster points of the sequence $\left\{\mathbf{z}_{k}\right\}$ are solutions of the (primal) problem (13) [47].

In the particular case when $g(\mathbf{u})=\mathbf{G u}$, where $\mathbf{G} \in \mathbb{R}^{d \times n}$, ALM/MM can be applied to problem (12) as follows,

$$
\begin{array}{ll}
\min _{\substack{\mathbf{u} \in \mathbb{R}^{n}, \mathbf{v} \in \mathbb{R}^{d} \\
\text { subject to }}} & f_{1}(\mathbf{u})+f_{2}(\mathbf{v}) \\
\mathbf{G} \mathbf{u}=\mathbf{v} .
\end{array}
$$

Problem (15) can be written in the form (13) using the following definitions:

$$
\mathbf{z}=\left[\begin{array}{c}
\mathbf{u} \\
\mathbf{v}
\end{array}\right], \quad \mathbf{b}=\mathbf{0}, \quad \mathbf{A}=\left[\begin{array}{ll}
-\mathbf{G} & \mathbf{I}
\end{array}\right]
$$

and

$$
E(\mathbf{z})=f_{1}(\mathbf{u})+f_{2}(\mathbf{v}) .
$$

With these definitions in place, Steps 3 and 4 of the ALM/MM (version II) can be written as follows:

$$
\begin{aligned}
\left(\mathbf{u}_{k+1}, \mathbf{v}_{k+1}\right) \in \arg \min _{\mathbf{u}, \mathbf{v}} & f_{1}(\mathbf{u})+f_{2}(\mathbf{v})+ \\
& \frac{\mu}{2}\left\|\mathbf{G u}-\mathbf{v}-\mathbf{d}_{k}\right\|_{2}^{2} \\
\mathbf{d}_{k+1}= & \mathbf{d}_{k}+\mathbf{G u}_{k+1}-\mathbf{v}_{k+1}
\end{aligned}
$$

The minimization problem (18) is not trivial since, in general, it involves non-separable quadratic and possibly non-smooth terms. A natural way to address (18) is to use a non-linear block-Gauss-Seidel (NLBGS) technique, in which (18) is solved by alternatingly minimizing it with respect to $\mathbf{u}$ and 
$\mathbf{v}$, while keeping the other variable fixed. Experimental evidence in [10] suggests that an efficient algorithm is obtained by running just one NLBGS step. It turns out that the resulting algorithm is an instance of the socalled alternating direction method of multipliers (ADMM) [7], which works as follows:

\section{Algorithm $A D M M$}

1. Set $k=0$, choose $\mu>0, \mathbf{v}_{0}$, and $\mathbf{d}_{0}$.

2. repeat

3. $\quad \mathbf{u}_{k+1} \in \arg \min _{\mathbf{u}} f_{1}(\mathbf{u})+\frac{\mu}{2}\left\|\mathbf{G u}-\mathbf{v}_{k}-\mathbf{d}_{k}\right\|_{2}^{2}$

4. $\quad \mathbf{v}_{k+1} \in \arg \min _{\mathbf{v}} f_{2}(\mathbf{v})+\frac{\mu}{2}\left\|\mathbf{G u}_{k+1}-\mathbf{v}-\mathbf{d}_{k}\right\|_{2}^{2}$

5. $\quad \mathbf{d}_{k+1}=\mathbf{d}_{k}+\mathbf{G u}_{k+1}-\mathbf{v}_{k+1}$

6. $\quad k \leftarrow k+1$

7. until stopping criterion is satisfied.

\subsection{Reconstruction}

We now apply the framework described in the previous sub-section to address the non-separable problem (10) of reconstructing the image from speckled and incomplete observations. We perform a variable splitting by introducing an auxilliary variable $\mathbf{u}$ to serve as the argument of the TV term, leading to a problem with the variables $(\mathbf{x}, \mathbf{f}, \mathbf{u})$ and two constraints,

$$
\min _{\mathbf{x}, \mathbf{f}, \mathbf{u}} \sum_{i}\left(\frac{y_{i}^{2}}{2} e^{-f_{i}}+f_{i}\right)+\frac{\lambda}{2} T V(\mathbf{u}), \quad \text { subject to } \quad \mathbf{A x}=e^{\mathbf{f}}, \quad \mathbf{x}=\mathbf{u} .
$$

Recall that our original data fidelity term is (9). Problem (20) is equivalent to,

$$
\min _{\mathbf{x}, \mathbf{u}, \mathbf{v}} \sum_{i}\left(\frac{y_{i}^{2}}{2 v_{i}}+\log v_{i}\right)+\frac{\lambda}{2} T V(\mathbf{u}), \quad \text { subject to } \quad \mathbf{A x}=\mathbf{v}, \quad \mathbf{x}=\mathbf{u} .
$$

It will be clear later in this sub-section that the formulation (20) has advantages.

Applying the AL/ADMM framework from algorithm ADMM, the problem to be minimized at iteration $k$ is,

$$
\min _{\mathbf{x}, \mathbf{f}, \mathbf{u}} \sum_{i}\left(\frac{y_{i}^{2}}{2} e^{-f_{i}}+f_{i}\right)+\frac{\lambda}{2} \operatorname{TV}(\mathbf{u})+\frac{\mu_{f}}{2}\left\|\mathbf{A x}-e^{\mathbf{f}}-\mathbf{d}_{f}^{k}\right\|_{2}^{2}+\frac{\mu_{u}}{2}\left\|\mathbf{x}-\mathbf{u}-\mathbf{d}_{u}^{k}\right\|_{2}^{2},
$$


where $\mu_{f}, \mu_{u}>0$ are the two penalty parameters corresponding to the two constraints, with the respective Bregman update vectors $\mathbf{d}_{f}$ and $\mathbf{d}_{u}$.

As in the case of (15), this problem is split into three problems at each iteration by gathering all the terms in each variable $\mathbf{x}, \mathbf{f}$ and $\mathbf{u}$, and solving for each by keeping the others fixed. The ADMM algorithm iterates between minimizing the objective function in (22) with respect to each of $\mathbf{x}, \mathbf{f}$ and $\mathbf{u}$, leading to a Gauss-Seidel process which at iteration $k$ is summarized as,

$$
\begin{aligned}
\mathbf{f}^{k+1} & =\arg \min _{\mathbf{f}} \sum_{i}\left(\frac{y_{i}^{2}}{2} e^{-f_{i}}+f_{i}\right)+\frac{\mu_{f}}{2}\left\|\mathbf{A} \mathbf{x}^{\mathbf{k}}-e^{\mathbf{f}^{\mathbf{k}}}-\mathbf{d}_{f}^{k}\right\|_{2}^{2}, \\
\mathbf{u}^{k+1} & =\arg \min _{\mathbf{u}} \frac{\lambda}{2} \operatorname{TV}(\mathbf{u})+\frac{\mu_{u}}{2}\left\|\mathbf{x}^{k}-\mathbf{u}-\mathbf{d}_{u}^{k}\right\|_{2}^{2}, \\
\mathbf{x}^{k+1} & =\arg \min _{\mathbf{x}} \frac{\mu_{f}}{2}\left\|\mathbf{A} \mathbf{x}-e^{\mathbf{f}^{\mathbf{k}+\mathbf{1}}}-\mathbf{d}_{f}^{k}\right\|_{2}^{2}+\frac{\mu_{u}}{2}\left\|\mathbf{x}-\mathbf{u}^{k+1}-\mathbf{d}_{u}^{k}\right\|_{2}^{2} \\
\mathbf{d}_{f}^{k+1} & =\mathbf{d}_{f}^{k}-\left(\mathbf{A} \mathbf{x}^{\mathbf{k}+\mathbf{1}}-e^{\mathbf{f}^{\mathbf{k}+\mathbf{1}}}\right) \\
\mathbf{d}_{u}^{k+1} & =\mathbf{d}_{u}^{k}-\left(\mathbf{x}^{\mathbf{k}+\mathbf{1}}-\mathbf{u}^{k+1}\right) .
\end{aligned}
$$

Problem (24) can be recognized to be a TV regularized quadratic minimization problem. However, since the TV function does not have an associated Moreau Proximal mapping [48] which yields an exact solution to the $\ell_{2}+\mathrm{TV}$ denoising problem, step (33) is solved approximately using Chambolle's algorithm [6].

The objective function in (23) is separable for each voxel $f_{i}$, and can be decomposed into $m$ problems,

$$
f_{i}^{k+1}=\arg \min _{f_{i}} \frac{y_{i}^{2}}{2} e^{-f_{i}}+f_{i}+\frac{\mu_{f}}{2}\left(\left(\mathbf{A} \mathbf{x}^{k}\right)_{i}-e^{f_{i}}-\left(d_{f}\right)_{i}^{k}\right)^{2} .
$$

This is a convex function and can be solved using a few iterations of Newton's method, as in [25]. Using the formulation (21) would have led to a non-convex function,

$$
v_{i}^{k+1}=\arg \min _{v_{i}} \frac{y_{i}^{2}}{2 v_{i}}+\log v_{i}+\frac{\mu_{f}}{2}\left(\left(\mathbf{A} \mathbf{x}^{k}\right)_{i}-v_{i}-\left(d_{f}\right)_{i}^{k}\right)^{2} .
$$

The step (23) is the only point where the exponential or logarithmic relation between the partial observations $\mathbf{A x}$ and $\mathbf{f}$ or $\mathbf{u}$ comes into the picture. Because the ADMM algorithm solves alternatingly with respect to each variable keeping all others fixed, the update steps

$$
\mathbf{d}_{f}^{k+1}=\mathbf{d}_{f}^{k}-\left(\mathbf{A} \mathbf{x}^{\mathbf{k}+\mathbf{1}}-e^{\mathbf{f}^{\mathbf{k}+\mathbf{1}}}\right),
$$


and

$$
\mathbf{d}_{f}^{k+1}=\mathbf{d}_{f}^{k}-\left(\mathbf{A} \mathbf{x}^{\mathbf{k}+\mathbf{1}}-\mathbf{v}^{\mathbf{k}+\mathbf{1}}\right)
$$

are equivalent.

Since the objective function in subproblem (25) is a sum of least squares terms, the minimizer at each iteration is,

$$
\begin{aligned}
x_{i}^{k+1} & =\arg \min _{x_{i}} \frac{\mu_{f}}{2}\left\|\mathbf{A} \mathbf{x}-e^{\mathbf{f}^{\mathbf{k}+\mathbf{1}}}-\mathbf{d}_{f}^{k}\right\|_{2}^{2}+\frac{\mu_{u}}{2}\left\|\mathbf{x}-\mathbf{u}^{k+1}-\mathbf{d}_{u}^{k}\right\|_{2}^{2} \\
& =\left(\mu_{f} \mathbf{A}^{T} \mathbf{A}+\mu_{u} \mathbf{I}\right)^{-1}\left(\mu_{f}\left(e^{\mathbf{f}^{\mathbf{k}+\mathbf{1}}}+\mathbf{d}_{f}^{k}\right)+\mu_{u}\left(\mathbf{u}^{k+1}+\mathbf{d}_{u}^{k}\right)\right) .
\end{aligned}
$$

This step can be computed efficiently because the structure of $\mathbf{A}$ implies that $\left(\mu_{f} \mathbf{A}^{T} \mathbf{A}+\mu_{u} \mathbf{I}\right)^{-1}$ is a diagonal matrix. Matrix $\mathbf{A}$ is an $m \times n$ binary matrix, with $m<n$, which is a subset of the rows of an identity matrix. Due to this particular structure, the product $\mathbf{A}^{T} \mathbf{A}$ is a diagonal matrix with diagonal elements equal to 1 at the positions corresponding to observed pixels, and 0 otherwise. Therefore the matrix $\left(\mu_{f} \mathbf{A}^{T} \mathbf{A}+\mu_{u} \mathbf{I}\right)$ is a diagonal, invertible matrix with diagonal elements equal to $\mu_{f}+\mu_{u}$ at the positions corresponding to observed pixels, and $\mu_{u}$ otherwise. Therefore, the inverse of this term is also diagonal, and the matrix multiplication in (30) is computed by a weighted element-wise multiplication of the residual term $\mu_{f}\left(e^{\mathbf{f}^{\mathrm{k}+\mathbf{1}}}+\mathbf{d}_{f}^{k}\right)+$ $\mu_{u}\left(\mathbf{u}^{k+1}+\mathbf{d}_{u}^{k}\right)$.

The proposed method is summarized in algorithm ADMM-Reconstruction.

\section{Algorithm ADMM-Reconstruction}

1. Set $k=0$, choose $\lambda, \mu_{f}, \mu_{u}>0, \mathbf{x}^{0}, \mathbf{f}^{0}, \mathbf{u}^{0}, \mathbf{d}_{f}^{0}$, and $\mathbf{d}_{u}^{0}$.

2. repeat

3 . Compute $\mathbf{f}^{k+1}$, by solving eq $(28)$ for $i=1, \ldots, m$, using Newton's method,

4. Compute $\mathbf{u}^{k+1}$, by solving eq (24) using Chambolle's algorithm,

5. $\quad x_{i}^{k+1}=\left(\mu_{f} \mathbf{A}^{T} \mathbf{A}+\mu_{u} \mathbf{I}\right)^{-1}\left(\mu_{f}\left(e^{\mathbf{f}^{\mathbf{k}+\mathbf{1}}}+\mathbf{d}_{f}^{k}\right)+\mu_{u}\left(\mathbf{u}^{k+1}+\mathbf{d}_{u}^{k}\right)\right)$,

6. $\quad \mathbf{d}_{f}^{k+1} \leftarrow \mathbf{d}_{f}^{k}-\left(\mathbf{A} \mathbf{x}^{\mathbf{k}+\mathbf{1}}-e^{\mathbf{f}^{\mathbf{k}+\mathbf{1}}}\right)$,

7. $\quad \mathbf{d}_{u}^{k+1} \leftarrow \mathbf{d}_{u}^{k}-\left(\mathbf{x}^{\mathbf{k}+\mathbf{1}}-\mathbf{u}^{k+1}\right)$,

8. $\quad k \leftarrow k+1$

9. until stopping criterion is satisfied.

Even though (24) and (23) are not solved exactly, the convergence conditions for ADMM [7] only require that their error sequences decrease monotonically and $\mu_{u}$ and $\mu_{f}$ be positive. 


\subsection{Denoising}

For denoising when there is no loss of pixels, we have $m=n$ and $\mathbf{A}=\mathbf{I}$. Therefore applying variable splitting to (5) leads to an optimization problem with only one constraint $\mathbf{f}=\mathbf{u}$,

$$
\min _{\mathbf{f}, \mathbf{u}} \sum_{i}\left(\frac{y_{i}^{2}}{2} e^{-f_{i}}+f_{i}\right)+\frac{\lambda}{2} T V(\mathbf{u}), \quad \text { subject to } \mathbf{f}=\mathbf{u} .
$$

It is straightforward to show that applying AL/ADMM to (32) leads to the Gauss-Seidel scheme,

$$
\begin{aligned}
\mathbf{u}^{k+1} & =\arg \min _{\mathbf{u}} \frac{\lambda}{2} T V(\mathbf{u})+\frac{\mu}{2}\left\|\mathbf{f}^{k}-\mathbf{u}-\mathbf{d}^{k}\right\|_{2}^{2}, \\
\mathbf{f}^{k+1} & =\arg \min _{\mathbf{f}} \sum_{i}\left(\frac{y_{i}^{2}}{2} e^{-f_{i}}+f_{i}\right)+\frac{\mu}{2}\left\|\mathbf{f}-\mathbf{u}^{k+1}-\mathbf{d}^{k}\right\|_{2}^{2}, \\
\mathbf{d}^{k+1} & =\mathbf{d}^{k}+\mathbf{u}^{k+1}-\mathbf{f}^{k+1},
\end{aligned}
$$

where $\mu>0$ is the penalty parameter and $\mathbf{d}$ is the Bregman update vector. As in the case of reconstruction, (33) is an $\ell_{2}+\mathrm{TV}$ denoising problem and solved using Chambolle's method, and (34) is solved using Newton's method.

\section{Experimental Results}

We evaluate the performance of the proposed method based on accuracy of reconstruction, and computational speed. To be able to evaluate the accuracy, we need to have access to a ground truth reference noiseless image. Since in practice, we do not have ground truth for real US images, we use synthetic examples in which known images are corrupted with Rayleigh multiplicative noise, to test our proposed method.

For the case of $2 \mathrm{D}$ reconstruction from partial observations, we compare our method pixel nearest neighbor (PNN) interpolation followed by RLTV, LPAMA/MV and DPAD[35]. This is because interpolation methods do not take into account the statistical model. For the 3D case, we compare the proposed method with PNN, which has been found to be the fastest interpolation method [31],[14], followed by denoising using 3D RLTV.

In the synthetic examples, the original noiseless image is multiplied with randomly generated Rayleigh distributed noise, and the denoised image is compared with the original, in terms of the reconstruction error. The error 
measure used was the Median Absolute Error (MAE) [29] which is defined as,

$$
\operatorname{MAE}=\frac{1}{N_{x}}\|\mathbf{x}-\hat{\mathbf{x}}\|_{1},
$$

where $\mathbf{x}$ is the ground truth image, $\hat{\mathbf{x}}$ is the estimate, $N_{x}$ is the number of pixels, and the $\ell_{1}$-norm, $\|\cdot\|_{1}$ is the sum of the absolute values of the difference vector.

All experiments were performed on MATLAB on a $2.4 \mathrm{GHz}$ Intel Xeon based Ubuntu Linux server, with 64 GB of RAM. The proposed method was run until the relative difference $\left\|\hat{\mathbf{x}}^{k+1}-\hat{\mathbf{x}}^{k}\right\|_{2} /\left\|\hat{\mathbf{x}}^{k}\right\|_{2}$ fell below $10^{-3}$.

The values of regularization parameters used were hand-tuned for each method for the synthetic examples, to obtain the best possible MAE. This is done because the different methods use different formulations, for example, with and without logarithmic transformation, and because we use denoising methods to despeckle interpolated images. For LPAMA/MV, the values of additional parameters used were the ones suggested in the authors' implementation.

\section{Choice of parameter values}

Experimentally, we observed that for values of the penalty parameter $\mu_{f}$ below 10, the algorithm encountered numerical instability. For the SheppLogan phantom image with $50 \%$ of pixels missing and $\lambda=1$, we varied the values of $\mu_{f}$ and $\mu_{u}$, and obtained the plots of the MAE and computational times shown in figure 1 . Each graph corresponds to a value of $\mu_{f}$. We can see from figure 1(a) that the error is relatively stable for $\mu_{f}$ between 20 and 100, and $\mu_{u}$ between 40 and 100 . The computational time does not vary much

for $\mu_{u}$ between 20 and 40, for values of $\mu_{f}$ above 60. As a trade-off, we use $\mu_{f}, \mu_{u}=50$ in our experiments.

\subsection{D Reconstruction}

We demonstrate the proposed algorithm on synthetic examples with the Shepp-Logan phantom, Lena, and cameraman images, with $50 \%$ of the pixels randomly discarded. The results of estimating the image and comparison with other methods is presented in table 1 . We see that the proposed method produces a lower MAE than interpolation followed by denoising.

The original Shepp-Logan phantom image is shown in figure 2(a). The observed image after multiplication with the noise and observation mask which randomly discards $50 \%$ of the pixels is shown in figure $2(\mathrm{~b})$. The 


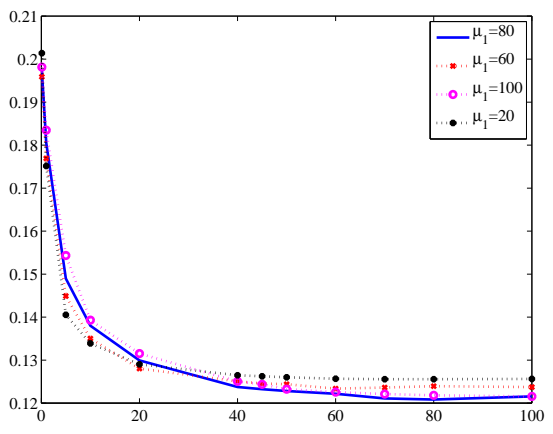

(a)

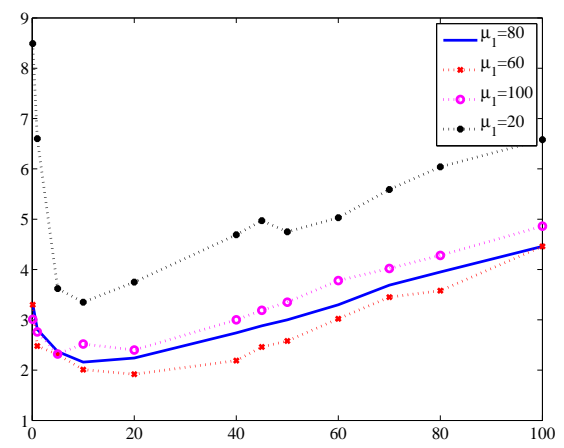

(b)

Figure 1: Varying the parameter values $\mu_{1}$ and $\mu_{2}$ for inpainting with the Shepp-Logan phantom, with $50 \%$ of the pixels randomly discarded : (a) median absolute error (MAE); (b) computational time.

estimate obtained using the proposed method is shown in figure 2(f). We present the plots of the evolution of the error sequences from the ADMM sequence in algorithm ADMM-Reconstruction in 3. Denoting the original noiseless image as $\mathbf{x}^{*}$, we see that the error sequences $\left\|\mathbf{f}^{(t)}-\log \left(\mathbf{A} \mathbf{x}^{*}\right)\right\|$ and $\left\|\mathbf{u}^{(t)}-\mathbf{x}^{*}\right\|$ decrease monotonically over time, which is in accordance with the convergence conditions from [7].

For the Lena and cameraman images, the original, observed, and estimated images are presented in figures 4 and 5 .

Table 1: 2D Inpainting. All images of size $256 \times 256$, with $50 \%$ of pixels randomly discarded.

\begin{tabular}{|l|c|c|c|c|c|}
\hline Image & Method & Iter.s & Time (sec.) & MAE & SSIM \\
\hline Shepp & Proposed & 16 & 1.77 & $\mathbf{0 . 1 2 2}$ & $\mathbf{0 . 9 9 5}$ \\
Logan & PNN+RLTV & 200 & 6.41 & 0.158 & 0.991 \\
Phantom & PNN+MV & 53 & $\mathbf{0 . 2 8}$ & 0.137 & 0.993 \\
& PNN+DPAD & 1000 & 22.45 & 0.123 & $\mathbf{0 . 9 9 5}$ \\
\hline \multirow{5}{*}{ Lena } & Proposed & 31 & 4.47 & $\mathbf{0 . 1 7}$ & $\mathbf{0 . 9 9 5}$ \\
& PNN+RLTV & 156 & 4.84 & 0.383 & 0.977 \\
& PNN+MV & 64 & $\mathbf{0 . 3 5}$ & 0.233 & 0.992 \\
& PNN+DPAD & 48 & 1.27 & 0.254 & 0.99 \\
\hline \multirow{5}{*}{ Cameraman } & Proposed & 30 & 4.31 & $\mathbf{0 . 0 7 8}$ & $\mathbf{0 . 9 9 9}$ \\
& PNN+RLTV & 200 & 6.36 & 0.347 & 0.979 \\
& PNN+MV & 70 & $\mathbf{0 . 4 3 3}$ & 0.252 & 0.99 \\
& PNN+DPAD & 100 & 2.75 & 0.289 & 0.986 \\
\hline
\end{tabular}




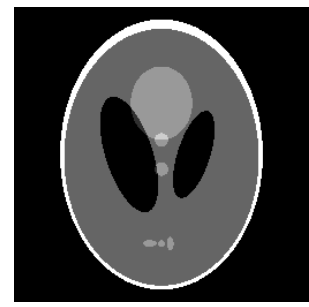

(a)

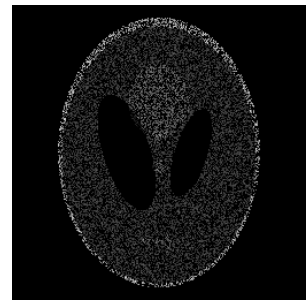

(b)

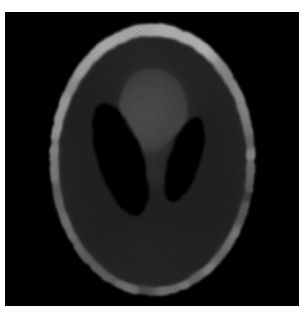

(e)

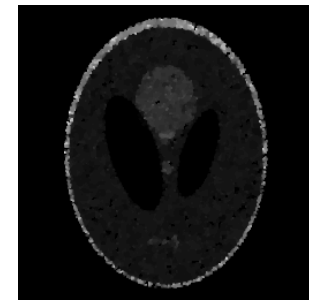

(c)

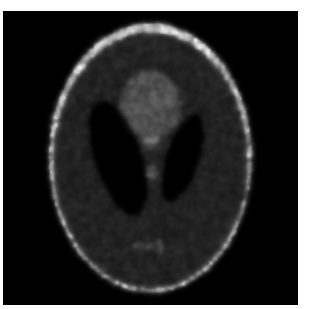

(f)

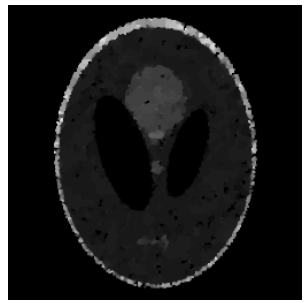

(d)

Figure 2: Inpainting with the Shepp-Logan phantom, with $50 \%$ of the pixels randomly discarded : (a) original; (b) observed image; estimates obtained using: (c) PNN+RLTV, (d) PNN+LPAMA/MV, (e) PNN+DPAD, (f) proposed method.

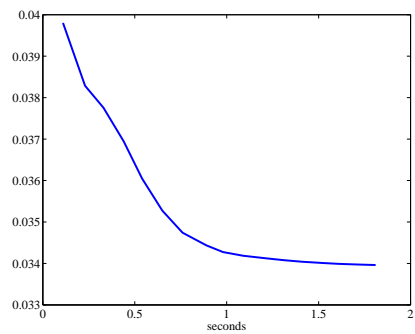

(a)

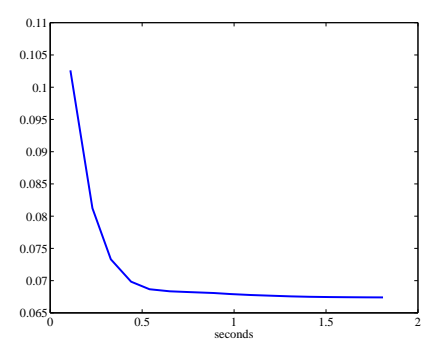

(b)

Figure 3: Evolution over time of ADMM error sequences for inpainting with the SheppLogan phantom, with $50 \%$ of the pixels randomly discarded : (a) $\left\|\mathbf{f}-\log \left(\mathbf{A} \mathbf{x}^{*}\right)\right\|$; (b) $\left\|\mathbf{u}-\mathbf{x}^{*}\right\|$.

\subsubsection{Different levels of missing pixels and Denoising}

We now run the proposed method against for different fractions of missing pixels, discarded at random. Notice that the denoising problem is the limit case, i.e., with the fraction of pixels missing equal to zero. Table 2 presents the results for different values of fraction of missing pixels, starting from the denoising case, for the Lena image. We can see that for denoising and lower 


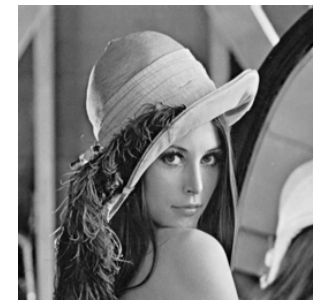

(a)

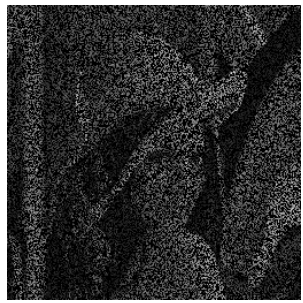

(b)

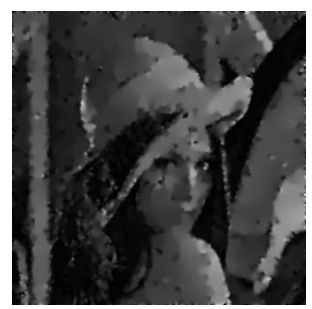

(e)

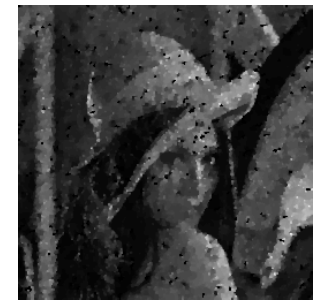

(c)

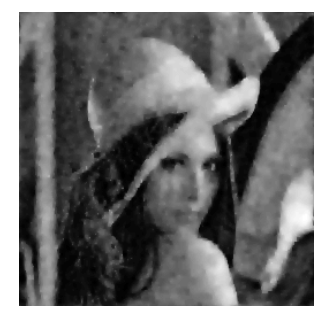

(f)

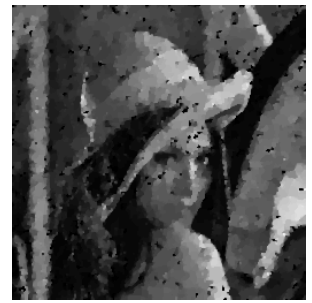

(d)

Figure 4: Inpainting with Lena, with $50 \%$ of the pixels randomly discarded : (a) original; (b) observed image; estimates obtained using: (c) PNN+RLTV, (d) PNN+LPAMA/MV, (e) PNN+DPAD, (f) proposed method.

fractions of missing pixels, PNN followed by denoising with LPAMA/MV produces the lowest MAE, but the for higher fractions, it is the proposed method which produces the lowest MAE.

The estimates obtained for denoising and reconstruction with $25 \%$ and $70 \%$ of the pixels missing are shown in figure 6 . Each row corresponds to one experiment. For example, the first row corresponds to denoising and shows the noisy image and the estimates. Note that for the second and third rows which correspond to reconstruction problems with $25 \%$ and $70 \%$ of the pixels missing, respectively, PNN was used before running the denoising methods.

\subsubsection{Real Ultrasound Images}

Figure 7 shows the RF images of the carotid artery (transversal and longitudinal), liver, and left ventricle. We use the random sampling pattern on all four images, with the results shown in figures 8, 9, 10, and 11. The radial sampling pattern is used on the images of the liver and left ventricle, with the results shown in figures 12 and 13.

Table 3 summarizes the results for inpainting with the random sampling pattern, and table 4 presents those for radial sampling. It can be seen that 


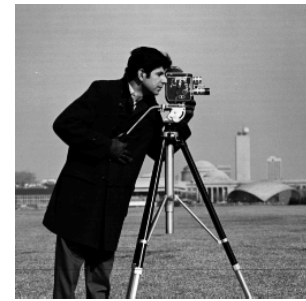

(a)

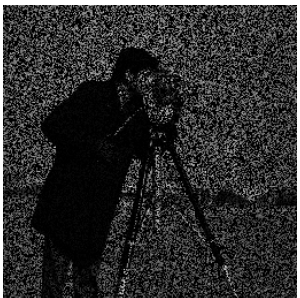

(b)

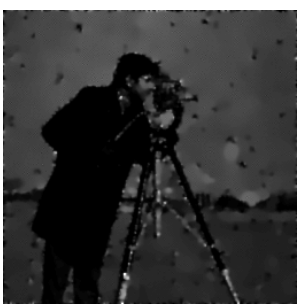

(e)

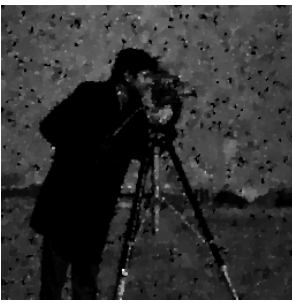

(c)

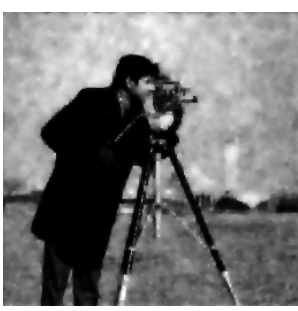

(f)

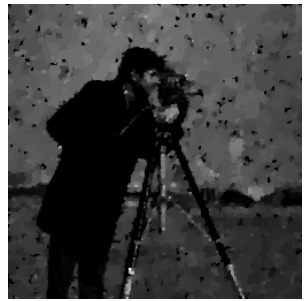

(d)

Figure 5: Inpainting with cameraman, with $50 \%$ of the pixels randomly discarded : (a) original; (b) observed image; estimates obtained using: (c) PNN+RLTV, (d) PNN+LPAMA/MV, (e) PNN+DPAD, (f) proposed method.

Table 2: Lena: 2D Inpainting, with varying fractions of pixels randomly discarded.

\begin{tabular}{|l|c|c|c|c|c|}
\hline Fraction missing & Method & Iter.s & Time (sec.) & MAE & SSIM \\
\hline \multirow{3}{*}{0 (denoising) } & ADMM & 19 & 2.31 & 0.22 & 0.993 \\
& RLTV & 276 & 8.35 & 0.116 & 0.998 \\
& LPAMA/MV & 112 & $\mathbf{0 . 5 7 9}$ & $\mathbf{0 . 0 7 7}$ & $\mathbf{0 . 9 9 9}$ \\
& DPAD & 7 & 0.25 & 0.14 & 0.997 \\
\hline \multirow{3}{*}{0.1} & Proposed & 35 & 4.29 & 0.245 & 0.991 \\
& PNN+RLTV & 328 & 9.61 & 0.375 & 0.978 \\
& PNN+LPAMA/MV & 46 & $\mathbf{0 . 2 4 8}$ & $\mathbf{0 . 1 5 3}$ & $\mathbf{0 . 9 9 6}$ \\
& PNN+DPAD & 123 & 3.31 & 0.254 & 0.99 \\
\hline \multirow{5}{*}{0.25} & Proposed & 26 & 2.75 & 0.238 & 0.991 \\
& PNN+RLTV & 275 & 7.89 & 0.374 & 0.978 \\
& PN+LPAMA/MV & 45 & $\mathbf{0 . 2 3 3}$ & $\mathbf{0 . 1 6 1}$ & $\mathbf{0 . 9 9 6}$ \\
0.5 & PNN+DPAD & 106 & 2.77 & 0.264 & 0.99 \\
\hline \multirow{5}{*}{0.7} & Proposed & 31 & 4.47 & $\mathbf{0 . 1 7}$ & $\mathbf{0 . 9 9 5}$ \\
& PNN+RLTV & 156 & 4.84 & 0.383 & 0.977 \\
& PNN+MV & 64 & $\mathbf{0 . 3 5}$ & 0.233 & 0.992 \\
& PNN+DPAD & 48 & 1.27 & 0.254 & 0.99 \\
\hline & Proposed & 47 & 4.3 & $\mathbf{0 . 1 9 4}$ & $\mathbf{0 . 9 9 4}$ \\
& PNN+RLTV & 200 & 6.39 & 0.421 & 0.972 \\
& PNN+LPAMA/MV & 93 & $\mathbf{0 . 5 8 5}$ & 0.357 & 0.98 \\
& PNN+DPAD & 27 & 0.83 & 0.302 & 0.986 \\
\hline
\end{tabular}




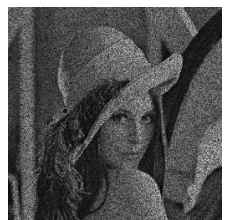

(a)

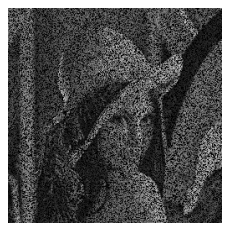

(f)

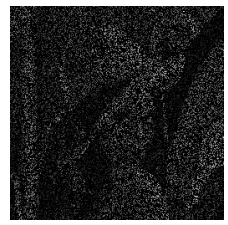

$(\mathrm{k})$

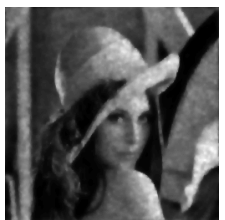

(b)

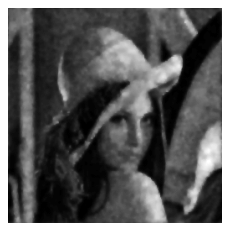

(g)

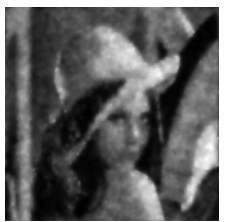

(l)

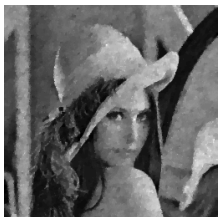

(c)

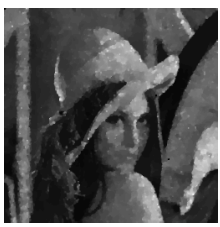

(h)

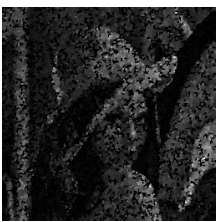

$(\mathrm{m})$

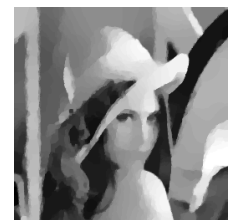

(d)

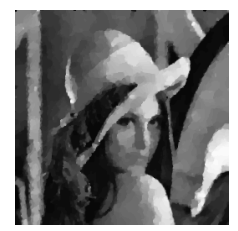

(i)

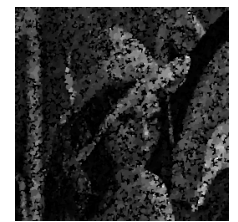

(n)

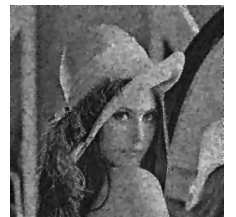

(e)

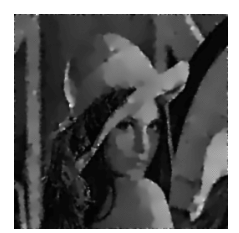

(j)

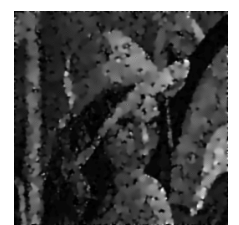

(o)

Figure 6: Denoising and inpainting with Lena, with different fractions of pixels randomly discarded. (a), (f), (k) noisy image with $0,25 \%$, and $70 \%$ of pixels missing, respectively; (b), (g), (l) respective estimates using the proposed method; (c), (h), (m) respective estimates using RLTV; (d), (i), (n) respective estimates using LPAMA/MV; (e), (j), (o) respective estimates using DPAD.

LPAMA/MV is the fastest method computationally, while DPAD reached the maximum number of iterations. Unlike in the case of the synthetic examples, we do not have the possibility to calculate the MAE and to optimize the parameters.

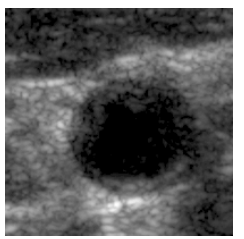

(a)

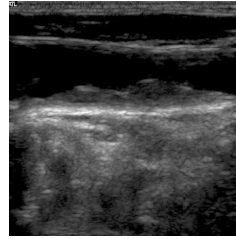

(b)

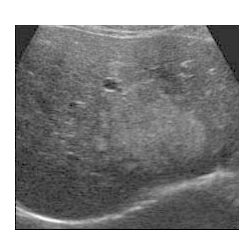

(c)

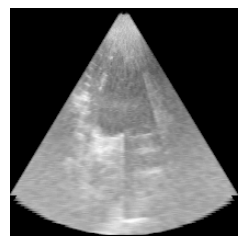

(d)

Figure 7: US radio frequency (RF) images: (a) carotid artery (transversal); (b) carotid artery (transversal); (c) liver; (c) PNN+RLTV, (d) left ventricle. 
Table 3: Inpainting with 2D ultrasound images, with $50 \%$ of pixels randomly discarded.

\begin{tabular}{|l|c|c|c|c|}
\hline Image & Size & Method & Iter.s & Time (sec.) \\
\hline \multirow{3}{*}{ carotid } & \multirow{3}{*}{$201 \times 201$} & Proposed & 89 & 6.97 \\
transversal & & PNN+RLTV & 241 & 4.46 \\
& & PNN+MV & 97 & 0.258 \\
& & PNN+DPAD & 100 & 1.95 \\
\hline \multirow{3}{*}{ carotid } & \multirow{3}{*}{$480 \times 464$} & Proposed & 60 & 27.64 \\
& & PNN+RLTV & 65 & 6.81 \\
& & PNN+MV & 92 & 1.488 \\
& & PNN+DPAD & 100 & 10.66 \\
\hline \multirow{3}{*}{ liver } & \multirow{3}{*}{$205 \times 229$} & Proposed & 165 & 15.44 \\
& & PNN+RLTV & 1322 & 29.08 \\
& & PNN+MV & 473 & 2.198 \\
& & PNN+DPAD & 1000 & 22.36 \\
\hline \multirow{3}{*}{ left } & \multirow{3}{*}{$208 \times 208$} & Proposed & 66 & 8.7 \\
ventricle & & PNN+RLTV & 289 & 5.1 \\
& & PNN+MV & 360 & 1.263 \\
& & PNN+DPAD & 1000 & 16.82 \\
\hline
\end{tabular}

Table 4: Inpainting with 2D ultrasound images, with radial sampling.

\begin{tabular}{|l|c|c|c|c|}
\hline Image & Size & Method & Iter.s & Time (sec.) \\
\hline \multirow{3}{*}{ liver } & \multirow{3}{*}{$205 \times 229$} & Proposed & 178 & 16.74 \\
& & PNN+RLTV & 889 & 18.7 \\
& & PNN+MV & 533 & 2.73 \\
& & PNN+DPAD & 1000 & 22.64 \\
\hline \multirow{3}{*}{ left } & \multirow{2}{*}{$208 \times 208$} & Proposed & 71 & 9.39 \\
ventricle & & PNN+RLTV & 466 & 8.26 \\
& & PNN+MV & 166 & 0.576 \\
& & PNN+DPAD & 1000 & 15.92 \\
\hline
\end{tabular}

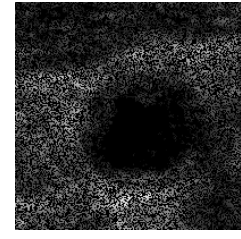

(a)

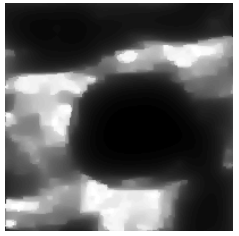

(b)

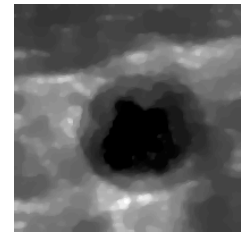

(c)

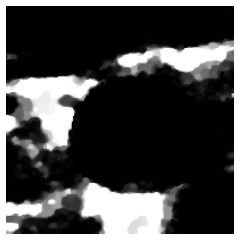

(d)

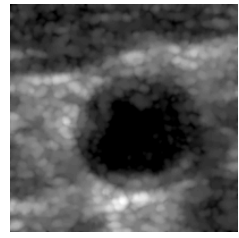

(e)

Figure 8: Inpainting with real US images: carotid artery (transversal). (a) observed image, estimates obtained using: (b) proposed method, (c) PNN+RLTV, (d) PNN+LPAMA/MV, (e) PNN+DPAD. 


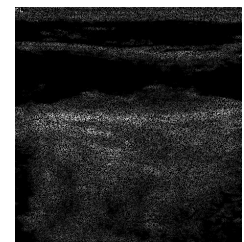

(a)

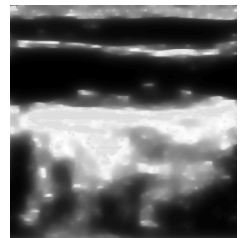

(b)

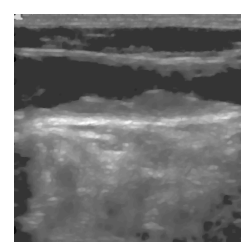

(c)

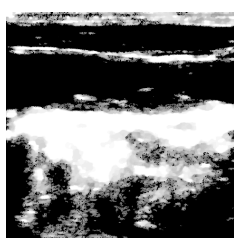

(d)

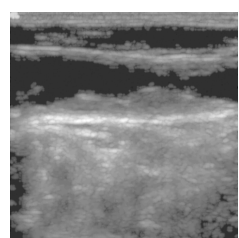

(e)

Figure 9: Inpainting with real US images: carotid artery (longitudinal). (a) observed image, estimates obtained using: (b) proposed method, (c) PNN+RLTV, (d) PNN+LPAMA/MV, (e) PNN+DPAD.

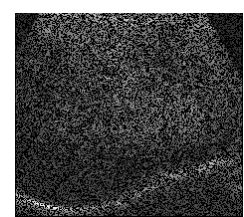

(a)

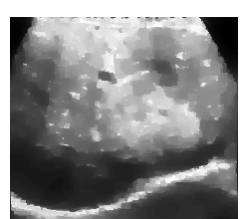

(b)

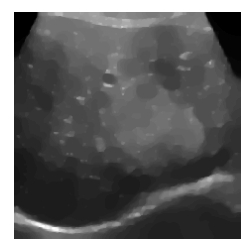

(c)

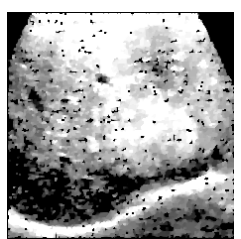

(d)

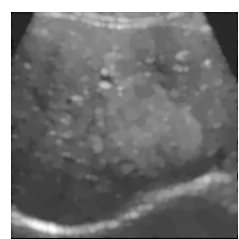

(e)

Figure 10: Inpainting with real US images: liver. (a) observed image, estimates obtained using: (b) proposed method, (c) PNN+RLTV, (d) PNN+LPAMA/MV, (e) PNN+DPAD.

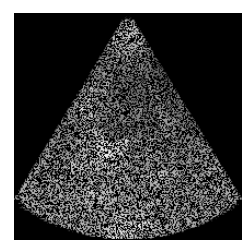

(a)

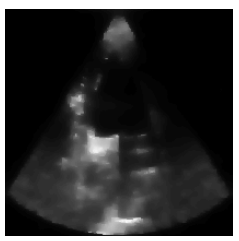

(b)

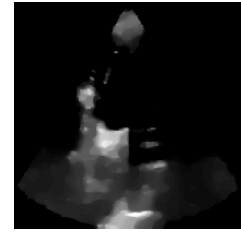

(c)

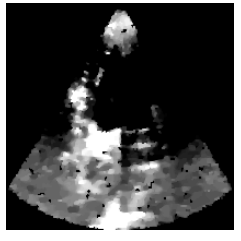

(d)

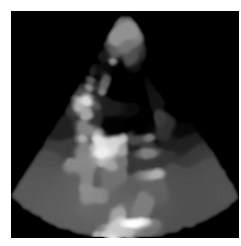

(e)

Figure 11: Inpainting with real US images: left ventricle. (a) observed image, estimates obtained using: (b) proposed method, (c) PNN+RLTV, (d) PNN+LPAMA/MV, (e) PNN+DPAD.

\subsection{D Reconstruction}

In the $3 \mathrm{D}$ case, as in the $2 \mathrm{D}$ one, we have access to the ground truth for the synthetic examples, but not for the real US data. By linear mechanical scanning, we mean that parallel and uniformly spaced slices are acquired along one axis. In freehand scanning, randomly positioned and oriented slices are acquired. Since LPAMA/MV and DPAD are 2D denoising methods, our comparison is against 3D RLTV after PNN interpolation. It was observed in [36] that PNN was the quickest interpolation method. 


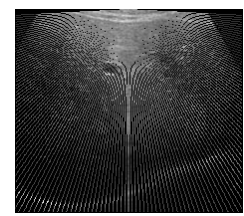

(a)

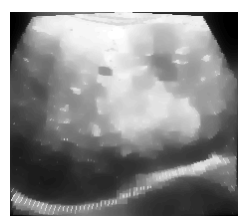

(b)

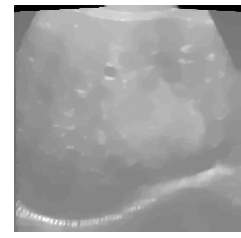

(c)

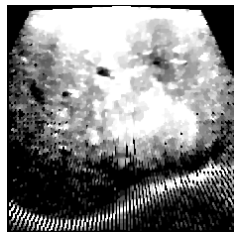

(d)

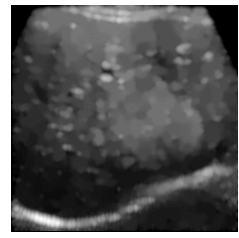

(e)

Figure 12: Inpainting with real US images, radial sampling: liver. (a) observed image, estimates obtained using: (b) proposed method, (c) PNN+RLTV, (d) PNN+LPAMA/MV, (e) PNN+DPAD.

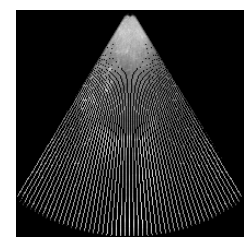

(a)

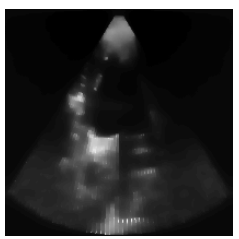

(b)

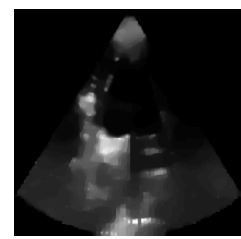

(c)

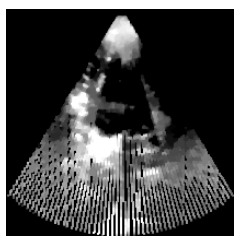

(d)

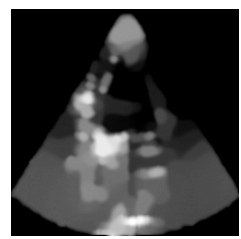

(e)

Figure 13: Inpainting with real US images, radial sampling: left ventricle. (a) observed image, estimates obtained using: (b) proposed method, (c) PNN+RLTV, (d) PNN+LPAMA/MV, (e) PNN+DPAD.

We use two synthetic volumes, a cylindrical tube with ones in the volume of the cylinder and zeros elsewhere and the 3D Shepp-Logan phantom, both of size $60 \times 60 \times 60$. Linear mechanical scanning is performed over the z-axis, by acquiring 30 equally spaced slices parallel to the xy-plane. This is shown in figures 14 and 16. Notice the gaps between lines in the yz- and xz- cross sectional views in the observed volumes, which correspond to unobserved slices. With freehand acquisition, 30 slices of size $60 \times 60$ oriented randomly were acquired. The resulting observed volumes and reconstructed estimates are presented in figures 15 and 17.

Table 5 compares the proposed method against PNN+RLTV in terms of the MAE and computation time, for both linear mechanical and freehand acquisitions. It can be seen from this table that the proposed method achieves a lower MAE in most of the experiments.

To illustrate our method with a real example, we reconstructed a volume from a set of 2D US images of the carotid artery, acquired transversally over a region of length $8 \mathrm{~cm}$, with equal spacing. There were 60 slices of size $255 \times 282$, each roughly corresponding to an area of $3.9 \times 4$ sq.cm. 


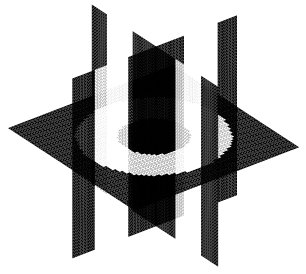

(a)

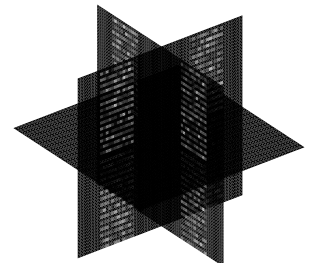

(b)

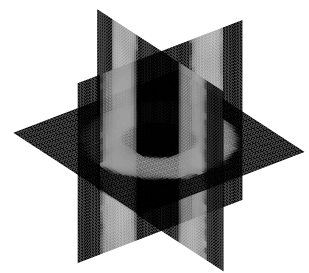

(d)

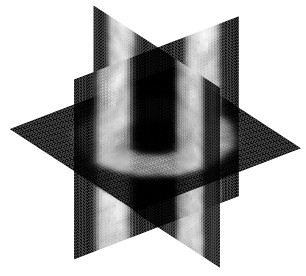

(c)

Figure 14: Synthetic example - 3D cylindrical tube, with linear mechanical acquisition along z-axis. 3D volumes of (a) original volume, (b) noisy and partial observations, (c) reconstructed volume using the proposed method, (d) reconstruction using PNN+RLTV.

Table 5: Comparison of 3D reconstruction.

\begin{tabular}{|l|c|c|c|c|c|}
\hline Experiment & Size & Acquisition & Method & CPU time (sec.) & MAE \\
\hline \multirow{3}{*}{ Cylinder } & \multirow{3}{*}{$60 \times 60 \times 60$} & linear & Proposed & $\mathbf{2 . 3 9}$ & 0.049 \\
& & & PNN+RLTV & 5.65 & $\mathbf{0 . 0 2 8}$ \\
\cline { 3 - 6 } & & freehand & Proposed & $\mathbf{1 . 5}$ & $\mathbf{0 . 2 9 5}$ \\
& & & PNN+RLTV & 5.69 & 0.309 \\
\hline \multirow{3}{*}{ Phantom } & \multirow{3}{*}{$64 \times 64 \times 64$} & linear & Proposed & 2.48 & $\mathbf{0 . 0 1 9}$ \\
& & & PNN+RLTV & $\mathbf{1 . 3 6}$ & 0.093 \\
\cline { 3 - 6 } & & freehand & Proposed & 6.21 & $\mathbf{0 . 0 3 7}$ \\
& & & PNN+RLTV & $\mathbf{5 . 4 7}$ & 0.039 \\
\hline carotid & $255 \times 282 \times 60$ & linear & Proposed & 43.01 & - \\
artery & & & RLTV 3D & 475.13 & - \\
\hline
\end{tabular}

The acquired B-mode images were first stacked together into the observed volume and then pre-processed to extract the RF image [40]. Fig 18 shows the observed stack of images and the reconstructed volumes. Because of the large dynamic range, the noisy RF and estimated images have been contrast enhanced for display. 


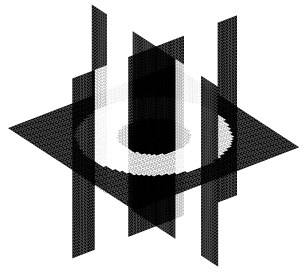

(a)

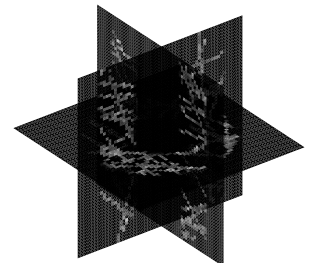

(b)

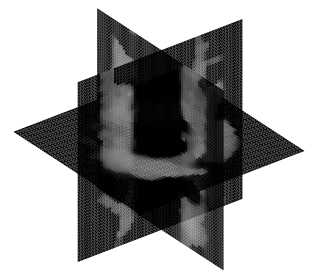

(d)

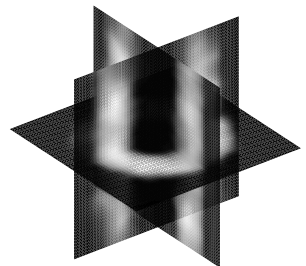

(c)

Figure 15: Synthetic example - 3D cylindrical, with freehand acquisition. 3D volumes of (a) original volume, (b) noisy and partial observations, (c) reconstructed volume using the proposed method, (d) reconstruction using PNN+RLTV.

The computation times are presented in table 5. Based on the results of the synthetic experiments, we can conclude that the proposed method achieves a performance comparable with that of RLTV after interpolation.

\section{Conclusions}

We have proposed an ADMM-based algorithm for image reconstruction with multiplicative speckle noise, assuming Rayleigh statistics. Experiments on synthetic and real US data showed that the proposed method offers a good balance between reconstruction accuracy and computation time. Only the pixel nearest neighbor interpolation method is computationally faster than our method, but does not take into account the statistical model and leads to a higher reconstruction error. For freehand scanning, the proposed method led to the lowest error in all experiments. The proposed method could therefore be a candidate for use as a recovery method for compressive sensing in US imaging and other situations where the Rayleigh multiplicative noise model is applicable. 


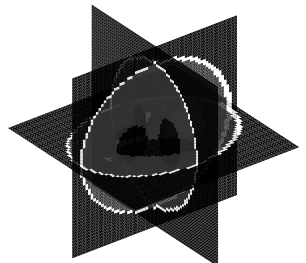

(a)

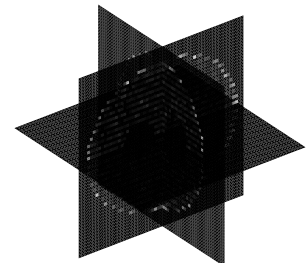

(b)

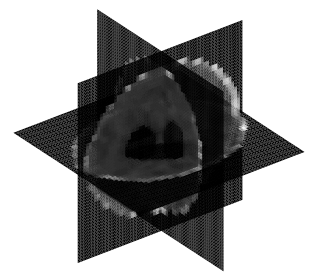

(d)

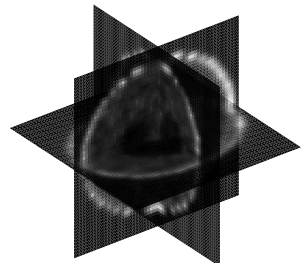

(c)

Figure 16: Synthetic example - 3D Shepp-Logan phantom, with linear mechanical acquisition along z-axis. 3D volumes of (a) original volume, (b) noisy and partial observations, (c) reconstructed volume using the proposed method, (d) reconstruction using PNN+RLTV.

Current and future work includes estimating the speckle field through the use of a non-sparse regularizer on the speckle component. Estimating the speckle in this way can lead to an estimate of the velocity of probe motion. In the case of linear mechanical scanning, this can help in estimating the slice position in the absence of a spatial locater. Another possible direction of research could be using the Kullback-Leibler (KL) divergence as a measure of the fidelity of the observations, and possibly to as a criterion for tuning the parameter values of the solvers. The KL divergence could also be used as a criterion for evaluating solvers in experiments with real data for which we are unable to calculate the reconstruction error.

\section{Acknowledgements}

This work was supported by the Fundação para a Ciência e Tecnologia (FCT), Portuguese Ministry of Science and Higher Education, through a Post-doctoral fellowship (contract no. SFRH/BPD/79011/2011) and FCT project (PEst-OE/EEI/LA0009/2013). 


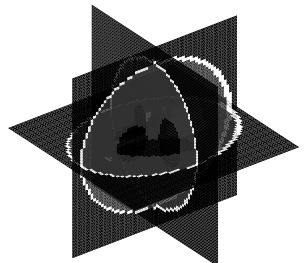

(a)

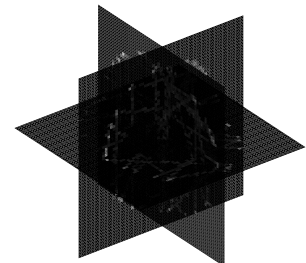

(b)

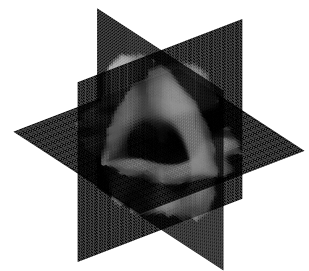

(d)

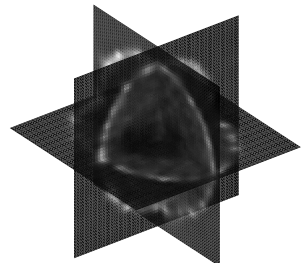

(c)

Figure 17: Synthetic example - 3D Shepp-Logan phantom, with freehand acquisition. 3D volumes of (a) original volume, (b) noisy and partial observations, (c) reconstructed volume using the proposed method, (d) reconstruction using PNN+RLTV.

\section{References}

1. Bertero M, Boccacci P. Introduction to inverse problems in imaging. Bristol, UK: IOP Publishing; 1998.

2. Candès E, Romberg J, Tao T. Stable signal recovery from incomplete and inaccurate information. Communications on Pure and Applied Mathematics 2005;59(8):1207-1233.

3. Donoho D. Compressed sensing. IEEE Transactions on Information Theory 2006;52(4):1289-1306.

4. Rudin L, Osher S, Fatemi E. Nonlinear total variation based noise removal algorithms. Physica D 1992;60:259-268.

5. Chan T, Esedoglu S, Park F, Yip A. Recent developments in total variation image restoration. Handbook of Mathematical Models in Computer Vision 2005;:17-30. 


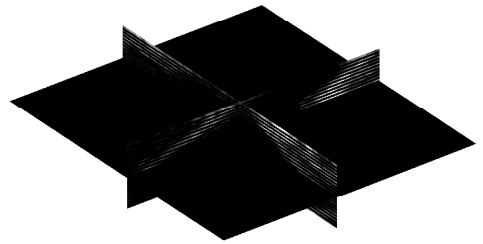

(a)

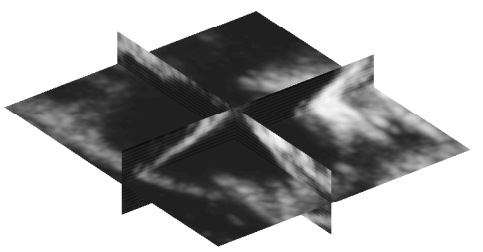

(b)

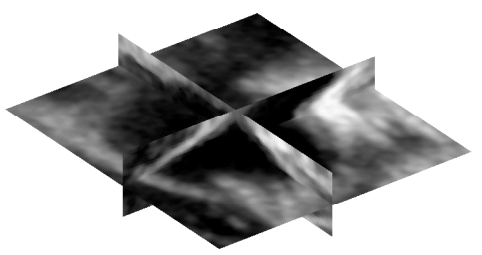

(c)

Figure 18: Carotid artery: (a) Observed RF volume; estimated using: (b) the proposed method, (c) PNN+ 3DRLTV.

6. Chambolle A. An algorithm for total variation minimization and applications. Journal of Mathematical Imaging and Vision 2004;20(1):89-97.

7. Eckstein J, Bertsekas D. On the DouglasRachford splitting method and the proximal point algorithm for maximal monotone operators. Mathematical Programming 1992;55(3):293-318.

8. Yin W, Osher S, Goldfarb D, Darbon J. Bregman iterative algorithms for $\ell_{1}$ minimization with applications to compressed sensing. SIAM Journal on Imaging Sciences 2008;1(1):143-168.

9. Wang Y, Yang J, Yin W, Zhang Y. A new alternating minimization algorithm for total variation image reconstruction. SIAM Journal on Imaging Sciences 2008;1(3):248-272.

10. Goldstein T, Osher S. The split Bregman method for $\ell_{1}$ regularized problems. SIAM Journal on Imaging Sciences 2009;2(2):323-343.

11. Afonso M, Bioucas-Dias J, Figueiredo M. Fast image recovery using variable splitting and constrained optimization. IEEE Trans on Im Proc 2010;19(9):2345-2356. 
12. Abbott JG, Thurstone F. Acoustic speckle: Theory and experimental analysis. Ultrasonic Imaging 1979;1(4):303-324.

13. Fenster A, Downey DB, Cardinal HN. Three-dimensional ultrasound imaging. Physics in Medicine and Biology 2001;46(5):R67.

14. Solberg O, Lindseth F, Torp H, Blake R, Nagelhus Hernes T. Freehand 3d ultrasound reconstruction algorithms-a review. Ultrasound in medicine $\&$ biology 2007;33(7):991-1009.

15. Coupé P, Hellier P, Azzabou N, Barillot C. 3d freehand ultrasound reconstruction based on probe trajectory. In: Medical Image Computing and Computer-Assisted Intervention. 2005, p. 597-604.

16. Solberg OV, Lindseth F, Bø LE, Muller S, Bakeng JBL, Tangen GA, et al. 3d ultrasound reconstruction algorithms from analog and digital data. Ultrasonics 2011;51(4):405 - 419.

17. Miller D, Lippert C, Vollmer F, Bozinov O, Benes L, Schulte D, et al. Comparison of different reconstruction algorithms for three-dimensional ultrasound imaging in a neurosurgical setting. The International Journal of Medical Robotics and Computer Assisted Surgery 2012;.

18. Wright S, Nowak R, Figueiredo M. Sparse reconstruction by separable approximation. IEEE Transactions on Signal Processing 2009; $\mathbf{5 7}(7): 2479-2493$.

19. Afonso M, Bioucas-Dias J, Figueiredo M. An augmented lagrangian based method for the constrained formulation of imaging inverse problems. IEEE Transactions on Image Processing 2011;20(3):681-695.

20. Dahl J, Hansen PC, Jensen SH, Jensen TL. Algorithms and software for total variation image reconstruction via first-order methods. Numerical Algorithms 2010;53(1):67-92.

21. Becker S, Bobin J, Candès E. NESTA: A fast and accurate first-order method for sparse recovery. SIAM Journal on Imaging Analysis 2011; 4:1-39.

22. Lustig M, Donoho D, Pauly J. Sparse mri: The application of compressed sensing for rapid $\mathrm{mr}$ imaging. Magnetic Resonance in Medicine 2007;58(6):1182-1195. 
23. Seabra J, Xavier J, Sanches J. Convex ultrasound image reconstruction with log-euclidean priors. In: Engineering in Medicine and Biology Society, EMBS'2008. IEEE; 2008, p. 435-438.

24. Seabra JCR. Medical Ultrasound B-Mode Modeling, De-speckling and Tissue Characterization Assessing the Atherosclerotic Disease. Ph.D. thesis; Instituto Superior Técnico; 2011.

25. Bioucas-Dias J, Figueiredo M. Multiplicative noise removal using variable splitting and constrained optimization. Image Processing, IEEE Transactions on 2010;19(7):1720 -1730.

26. Yun S, Woo H. A new multiplicative denoising variational model based on $\mathrm{m}$-th root transformation. Image Processing, IEEE Transactions on 2012;21(5):2523-2533.

27. Woo H, Yun S. Alternating minimization algorithm for speckle reduction with a shifting technique. Image Processing, IEEE Transactions on 2012;21(4):1701-1714.

28. Yu C, Zhang C, Xie L. A multiplicative nakagami speckle reduction algorithm for ultrasound images. Multidim Sys and Sig Proc 2012;:115 .

29. Durand S, Fadili J, Nikolova M. Multiplicative noise removal using 11 fidelity on frame coefficients. J Math Imaging Vis 2010;36(3).

30. Dong Y, Zeng T. A convex variational model for restoring blurred images with multiplicative noise. SIAM Journal on Imaging Sciences 2013; 6(3):1598-1625.

31. Rohling R, Gee A, Berman L. A comparison of freehand 3d ultrasound reconstruction techniques. Med Imag Analysis 1999;3(4):339 - 359.

32. Gobbi DG, Peters TM. Interactive intra-operative 3d ultrasound reconstruction and visualization. In: Medical Image Computing and Computer-Assisted Intervention; vol. 2489. 2002, p. 156-163.

33. Toonkum P, Suwanwela NC, Chinrungrueng C. Reconstruction of 3d ultrasound images based on cyclic regularized savitzky-golay filters. Ultrasonics 2011;51(2):136 - 147. 
34. Barratt D, Ariff B, Humphries K, Thom S, Hughes A. Reconstruction and quantification of the carotid artery bifurcation from 3-d ultrasound images. Medical Imaging, IEEE Transactions on 2004;23(5):567-583.

35. Aja-Fernández S, Alberola-López C. On the estimation of the coefficient of variation for anisotropic diffusion speckle filtering. Image Processing, IEEE Transactions on 2006;15(9):2694-2701.

36. Afonso MV, Sanches JMR. A total variation based reconstruction algorithm for 3d ultrasound. In: 6th Iberian Conference on Pattern Recognition and Image Analysis (IbPRIA). Funchal, Madeira, Portugal; 2013,

37. Masnou S, Morel JM. Level lines based disocclusion. In: Image Processing, 1998. ICIP 98. Proceedings. 1998 International Conference on. IEEE; 1998, p. 259-263.

38. Bertalmio M, Sapiro G, Caselles V, Ballester C. Image inpainting. In: Proceedings of the 27th Annual Conference on Computer Graphics and Interactive Techniques. ACM Press/Addison-Wesley Publishing Co.; 2000, p. 417-424.

39. Shen J, Chan TF. Mathematical models for local nontexture inpaintings. SIAM Journal on Applied Mathematics 2002;62(3):1019-1043.

40. Seabra J, Sanches J. Modeling log-compressed ultrasound images for radio frequency signal recovery. In: Engineering in Medicine and Biology Society. 2008, .

41. Rodrigues IC, Sanches JMR. Convex total variation denoising of poisson fluorescence confocal images with anisotropic filtering. IEEE Trans on Image Processing 2011;20(1):146-160.

42. Daubechies I, Defrise M, De Mol C. An iterative thresholding algorithm for linear inverse problems with a sparsity constraint. Communications on Pure and Applied Mathematics 2004;57(11):1413-1457.

43. Nocedal J, Wright S. Numerical optimization. Springer verlag; second ed.; 2006. 
44. Hestenes M. Multiplier and gradient methods. Journal of optimization theory and applications 1969;4(5):303-320.

45. Powell M. Optimization; chap. " A method for nonlinear constraints in minimization problems". New York: Academic Press; 1969, p. 283-298.

46. Bazaraa M, Sherali H, Shetty C. Nonlinear programming: theory and algorithms. New York: John Wiley and Sons; 1993.

47. Iusem A. Augmented Lagrangian methods and proximal point methods for convex optimization. Investigación Operativa 1999;8:11-49.

48. Combettes P, Wajs V. Signal recovery by proximal forward-backward splitting. SIAM Journal on Multiscale Modeling and Simulation 2006; 4(4):1168-1200. 
Manya Afonso finished his PhD at Instituto Superior Tecnico in 2011, while being a researcher at the Instituto de Telecomunicacoes, Lisboa. He is currently a Post-doc at the Instituto de Sistemas e Robotica in Instituto Superior Tecnico.

He previously received the Bachelor of Engineering degree in Electronics and Telecommunication Engineering from Goa University, India in 2003 and Master of Technology in Communication Engineering from the Indian Institute of Technology Delhi in 2005.

Manya Afonso's research interests include image processing and analysis, inverse problems, optimization, machine learning, computer vision, and video surveillance. 
Joao Miguel Raposo Sanches received the E.E., M.Sc., and Ph.D. degrees from the Technical University of Lisbon, Portugal, in 1991, 1996, and 2003, respectively.

Currently, he is Associate Professor (with 'aggregação') in the Electrical and Computer Engineering Department, Instituto Superior Técnico, Lisbon (IST), and a Researcher at the Institute for Systems and Robotics (ISR). He has taught in the area of signal processing and control. His main research interests are in biomedical engineering, namely in biomedical imaging processing and physiological modeling of biological systems with

several publications in the field. His research activity is mainly in ultrasound (US), functional magnetic resonance imaging (fMRI), fluorescence confocal microscope (FCM) imaging and neurophysiology, mostly in collaboration with several groups from the Medical School at the Hospital de Santa Maria, Lisbon. Dr. Sances is a member of the IEEE Engineering in Medicine and Biology Society and Associate Member of the Bio Imaging and Signal Processing Technical Committee (BISP$\mathrm{TC})$ of the IEEE Signal Processing Society. He is also president of the Portuguese Association of Pattern Recognition, APRP. 
${ }^{*}$ Photo of the author(s)

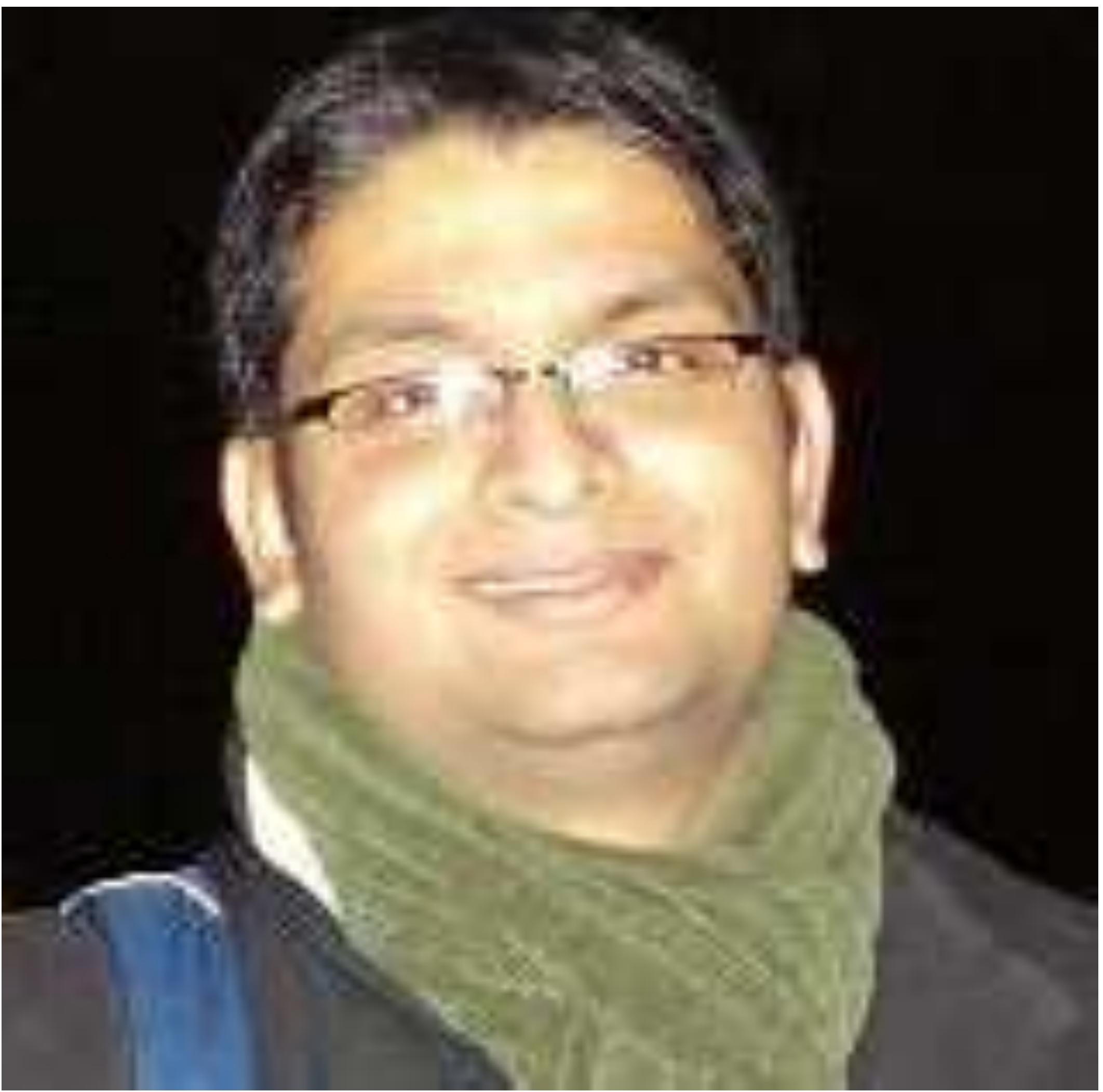

\section{(s)}

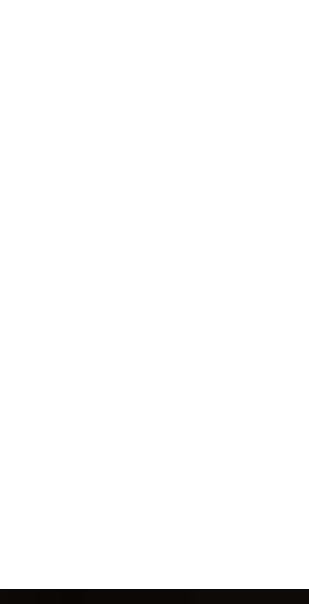
. (2) (2. 


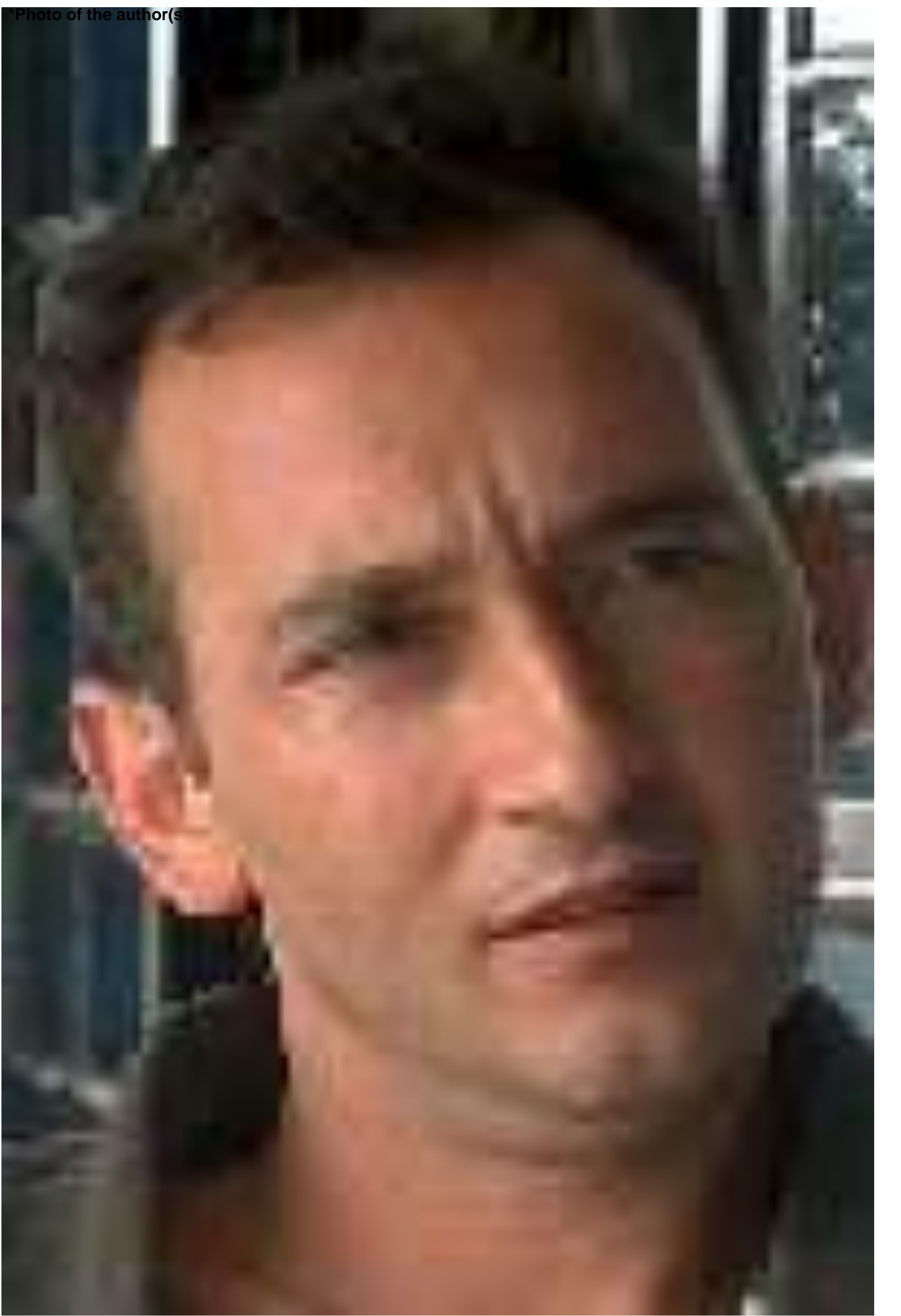

